\title{
Acute Epiploic Appendagitis in a COVID-19-Positive Patient With Antiphospholipid Syndrome
}

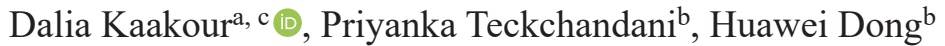

\begin{abstract}
As the coronavirus disease 2019 (COVID-19) pandemic is still underway, a range of clinical presentations and pathologies continue to present themselves in unexpected ways. One such pathology is that of epiploic appendagitis, an uncommon and underdiagnosed cause of acute abdominal pain. We present the case of a 50-something-yearold male who presented with left lower quadrant abdominal pain in the setting of acute COVID-19 infection, found to have acute epiploic appendagitis. After persistent moderate to severe abdominal pain, epiploic appendagitis was diagnosed by computed tomography (CT) imaging findings. The patient was managed for his COVID-19 pneumonia over the course of his hospitalization, as well as conservatively managed with pain control measures for his epiploic appendagitis. This is the second reported case in the literature to the best of our knowledge that shares the case of acute epiploic appendagitis in a patient presenting with acute abdominal pain, who is also found to be COVID-19-positive. Procoagulant changes in coagulation pathways are found in patients with severe COVID-19, and contribute to venous thromboembolism in this patient population. Diagnosing and conservatively managing epiploic appendagitis will lead to decreasing misdiagnosis, preventing invasive or inappropriate treatments that may increase harm to patients, and more adequately understanding the complications associated with COVID-19.
\end{abstract}

Keywords: Epiploic appendagitis; COVID-19; Antiphospholipid syndrome

\section{Introduction}

Epiploic appendagitis is an uncommon and underdiagnosed

Manuscript submitted November 9, 2021, accepted November 19, 2021

Published online December 28, 2021

aDepartment of Internal Medicine, University of California, Irvine, The City Drive South City Tower, Suite 400, Orange, CA 92868, USA

bivision of Pulmonary Diseases \& Critical Care Medicine, Department of Internal Medicine, University of California, Irvine, Orange, CA 92868, USA

${ }^{\mathrm{c} C}$ Corresponding Author: Dalia Kaakour, Department of Internal Medicine, University of California, Irvine, The City Drive South City Tower, Suite 400, Orange, CA 92868, USA. Email: dkaakour@hs.uci.edu

doi: https://doi.org/10.14740/jocmr4632 cause of acute abdominal pain, affecting about 8.8 per 1 million people per year. Since this condition may be misdiagnosed as diverticulitis or appendicitis, this number is likely an underestimation [1]. It is caused by twisting, inflammation, or thrombosis of the epiploic appendages, predominantly adipose-containing ovoid structures that arise on the antimesenteric serosal surface of the colon connected by a vascular pedicle [2]. Although the presenting symptoms of the condition are nonspecific, patients with epiploic appendagitis most commonly present with localized abdominal pain, more commonly on the left, exacerbated by coughing, deep breathing, or stretching. Nausea, vomiting and loss of appetite are rare symptoms. Patients typically have a normal white blood cell (WBC) count, or mild leukocytosis. Signs and symptoms are self-limiting and rarely last more than one week [3-5]. It is now well-established that high D-dimer levels and procoagulant changes in coagulation pathways are found in patients with severe coronavirus disease 2019 (COVID-19) [6, 7]. This leads to an elevated rate of venous and arterial thrombotic events in this patient population [8-10].

\section{Case Report}

\section{Investigations}

A 50-something-year-old male with systemic lupus erythematosus, antiphospholipid syndrome complicated by chronic lower extremity deep venous thromboses (on coumadin, international normalized ratio (INR) goal 3.5 - 4.5 and inferior vena cava (IVC) filter), chronic pain, chronic obstructive pulmonary disease, paroxysmal atrial fibrillation, myocardial infarction status post percutaneous intervention with two drug eluting stents, hypertension, and hyperlipidemia, presented to our emergency department with chest pain, shortness of breath, and left lower quadrant abdominal pain. Patient's body mass index (BMI) was 26.9, and he was a current one pack per day smoker with 30-pack-year history.

The patient reported that he had been feeling worsening shortness of breath with non-productive intermittent cough for about one week. The night prior, he awoke from sleeping with new onset of chest pain in the center of his chest, which he described as sharp, non-radiating, 10/10 in severity, and constant. His symptoms persisted over the course of the next couple of hours. He endorsed ongoing generalized headache, subjective fever, ageusia, nausea, vomiting, bloating, severe left lower 
Table 1. Patient Lab Values

\begin{tabular}{ll}
\hline Troponin & Negative $\times 2$ \\
White blood cell $(\mathrm{WBC})$ & $8.8 \times 10^{3} / \mu \mathrm{L}$ \\
Lymphocytes & $8.0 \%$ \\
D-dimer & $1,360 \mathrm{ng} / \mathrm{mL}$ \\
BNP & $33 \mathrm{pg} / \mathrm{mL}$ \\
INR & 1.33 \\
PT & $15.8 \mathrm{~s}$ \\
PTT & $25.9 \mathrm{~s}$ \\
COVID-19 PCR & Positive \\
\hline
\end{tabular}

BNP: brain natriuretic peptide; INR: international normalized ratio; COVID-19: coronavirus disease 2019; PCR: polymerase chain reaction; PT: prothrombin time; PTT: partial thromboplastin time.

quadrant abdominal pain, and diarrhea. He stated that the abdominal pain started several days after he noticed the cough and shortness of breath. The abdominal pain was exacerbated by pressure or palpation to the left lower quadrant, and with significant movement. He had not had relief despite pain medications. He denied anosmia, diarrhea, blood in his stool or constipation.

Upon presentation, the patient's pulse oximeter oxygen saturation $\left(\mathrm{SpO}_{2}\right)$ was $99 \%$ on $4-\mathrm{L}$ nasal cannula. He was afebrile with otherwise stable vital signs. On physical exam, patient was anxious, breathing comfortably on nasal cannula, abdominal exam positive for diffuse tenderness to palpation, worst in the left lower quadrant, bowel sounds normoactive, abdomen soft and non-distended. Labs were without significant abnormalities (Table 1).

Chest X-ray showed low lung volumes and hypoventilation changes. Computed tomography angiography (CTA) pulmonary embolism (PE) study did not show evidence of acute pulmonary embolism, but did note some mild lung paraseptal and centrilobular emphysema with bilateral dependent atelectasis. The patient was trialed off oxygen, but desaturated to the high 80 s with symptomatic shortness of breath. He was

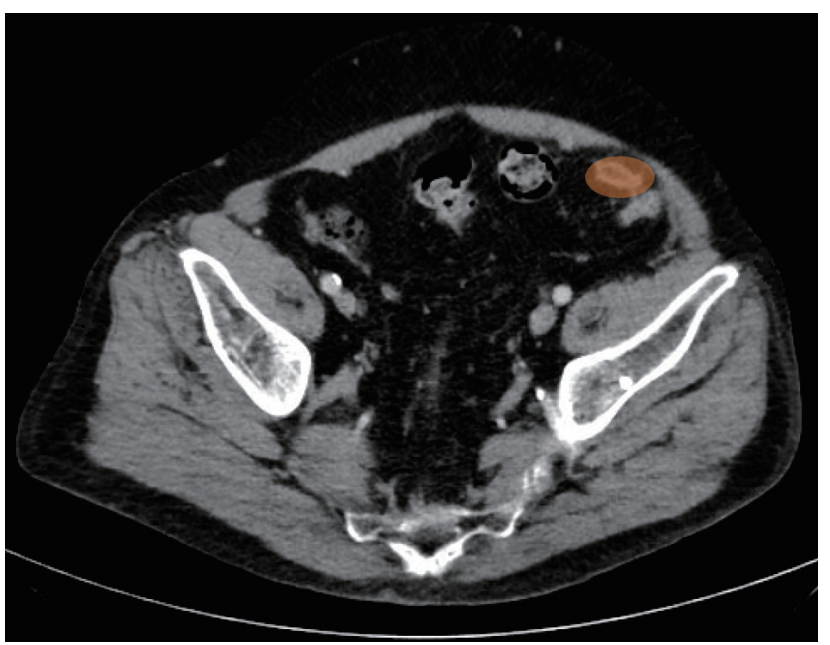

Figure 2. CT abdomen and pelvis from hospital day 2. The oval fat attenuation with soft tissue rim along the sigmoid colon mesentery is shown as the orange oval. CT: computed tomography.

self-proned which increased his abdominal pain, and admitted to the inpatient medicine team. Hospital course per respiratory status can be seen below in Figure 1.

Patient refused self-proning from this time and throughout his hospitalization, due to exacerbation of abdominal pain. Assuming the left lateral recumbent position would typically slightly improve his $\mathrm{O}_{2}$ saturation if he was desaturating.

\section{Diagnosis}

Meanwhile, patient continued to complain of abdominal pain, and he underwent computed tomography (CT) abdomen and pelvis on hospital day 2 which showed interval development of a $2.2 \times 1.2 \mathrm{~cm}$ oval fat attenuation with soft tissue rim along the sigmoid colon mesentery, new since January 11, 2019, consistent with epiploic appendagitis (Fig. 2). A diagnosis was

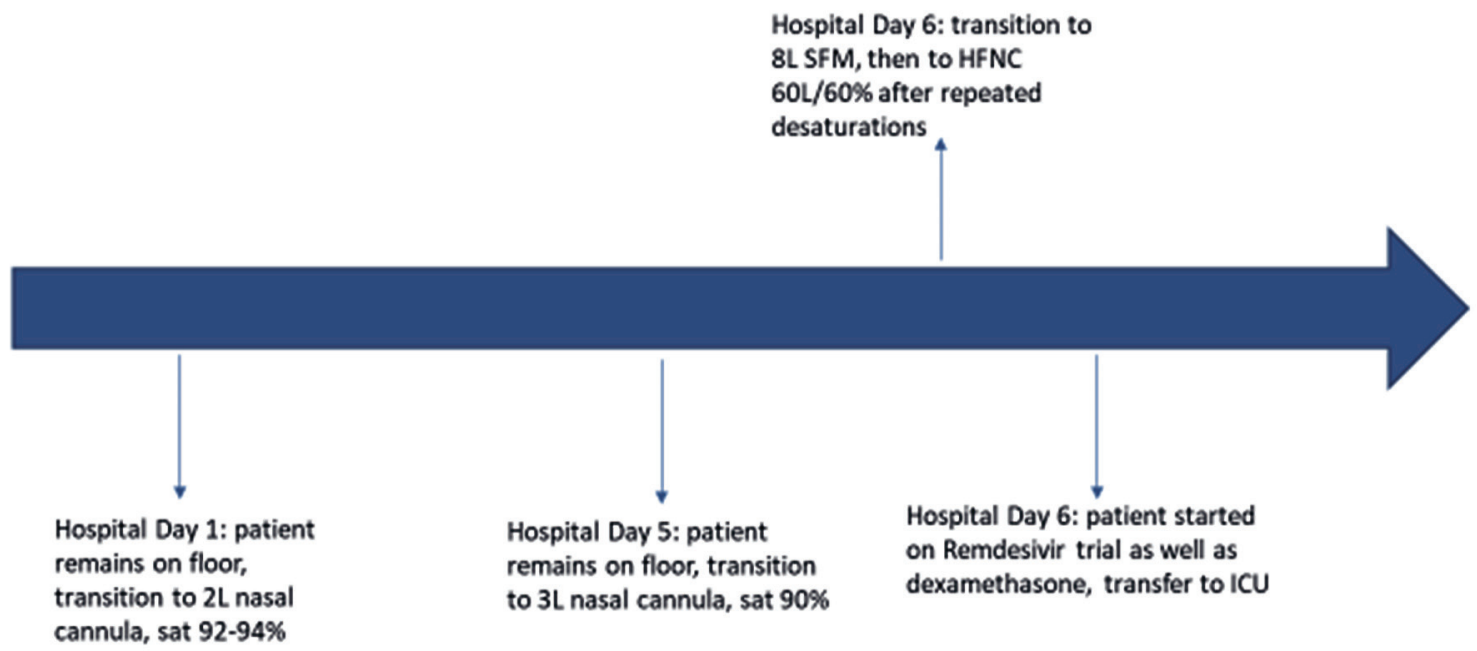

Figure 1. Timeline of respiratory status changes. SFM: simple face mask; HFNC: high-flow nasal cannula; ICU: intensive care unit. 
readily established based on clinical presentation, corroborated by CT imaging.

\section{Treatment}

The acute care surgery team was consulted, who recommended conservative management with an emphasis on pain control. Patient was also placed on $3.375 \mathrm{~g}$ of Zosyn for a 7-day course. Despite attempts at pain management with the help of the acute pain service, patient's abdominal pain continued throughout the course of his hospitalization.

\section{Follow-up and outcomes}

Patient went on to have a 24-day hospital course in total, primarily for the management of his COVID-19 pneumonia. He completed a 5-day course of remdesivir (200 mg loading dose once, following by 4 days of remdesivir $100 \mathrm{mg}$ daily) as well as a 10-day course of dexamethasone $6 \mathrm{mg}$ daily. Throughout this time, he remained on a pain control regimen, but still had unchanged abdominal pain, worst in the left lower quadrant and with palpation. He completed his course of Zosyn. He remained anticoagulated to his INR goal 3.5 - 4.5, for the most part, with the exception of during one episode of epistaxis, and also a large chest wall hematoma during which he suffered significant blood loss. The patient was eventually discharged to a transitional care hospital, on high-flow nasal cannula (HFNC) $40 \mathrm{~L} / 40 \%$, without resolution of his abdominal pain. To the best of our knowledge, the patient has not passed away, although he is not responsive to phone call attempts.

\section{Discussion}

Venous thromboembolism (VTE) is now a well-established complication of COVID-19, and early reports have suggested a VTE rate of approximately $27 \%$ in critically ill patients hospitalized with COVID-19 [8]. The high rates of VTE events in hospitalized and critically ill patients with COVID-19 occur despite standard anticoagulant thromboprophylaxis with unfractionated heparin or low-molecular-weight heparin (47/48 in the study of Ren et al and 20/22 in the study of Poissy et al $[11,12]$. Some studies, such as that by Llitjos et al have even found that VTE can occur in patients treated with therapeutic anticoagulation from admission [9].

This poses a clinical challenge in deciding the treatment of these patients, given the known and life-threatening risks associated with therapeutic anticoagulation, such as heparininduced thrombocytopenia, hemorrhagic complications, and heparin-associated osteoporosis. To date, there is no consensus on when and whether or not to provide therapeutic anticoagulation to critically ill COVID-19 patients $[13,14]$.

Furthermore, it is important to discuss antiphospholipid antibodies (aPLs) and COVID, especially in the case of this patient who had known antiphospholipid syndrome. The causality (if one exists) between aPL and COVID is unclear, al- though transient aPL elevations have been demonstrated in critically ill COVID patients. Even outside of the setting of COVID, aPL can rise transiently in patients with critical illness and various infections $[15,16]$. Other studies have contradicted this finding, showing that aPLs are not elevated in a population of 24 severely ill patients with COVID-19 and VTE [17].

Overall, the studies exploring the relationship between aPL and VTE in the setting of COVID are quite limited and the topic requires further investigation. Elucidating the pathophysiology of VTE in the setting of COVID will lead to the more appropriate treatment and decrease in morbidity and mortality associated with this complication [18].

\section{Learning points}

Epiploic appendagitis is an underdiagnosed and rare cause of acute abdominal pain. It can be caused by venous thrombosis, and should be considered in COVID-positive patients with abdominal pain, despite anticoagulation. Epiploic appendagitis has not yet been diagnosed as a known complication of COVID-19, and therefore its incidence and prevalence in these patients is unknown. Putting epiploic appendagitis on the radar as a cause of abdominal pain in COVID-19 patients has the potential to aid diagnosis, and to avoid misdiagnosis of the condition, avoiding clinical mismanagement in this patient population. Elucidating the causal factors for VTE in COVID-19, inside and outside of aPL will add to our understanding of the pathophysiology of COVID-19 and its complications, leading to improvements in our medical management of these deathly complications.

\section{Acknowledgments}

None to declare.

\section{Financial Disclosure}

The authors have no relevant financial interest to disclose. The authors did not receive any support from any organization for the submitted work.

\section{Conflict of Interest}

Case abstract was presented as a poster at the 2021 SGIM Northwest \& California-Hawaii Virtual Regional Meeting.

\section{Informed Consent}

Verbal informed consent was obtained from the patient.

\section{Author Contributions}

All authors have contributed substantially to the conception of 
the work, the drafting or revising of the work, have approved the final work to be published, and agree to be accountable for all aspects of the work. Dalia Kaakour was the lead in conception of the case report, as well as the primary author of this manuscript. Priyanka Teckchandani was involved in the conception of the case report, took part in writing portions, as well as significantly edited portions of this case report. Huawei Dong was also involved in conception of case report, and was editor.

\section{Data Availability}

The data supporting the findings of this study are available from the corresponding author upon reasonable request.

\section{References}

1. de Brito P, Gomez MA, Besson M, Scotto B, Huten N, Alison D. [Frequency and epidemiology of primary epiploic appendagitis on $\mathrm{CT}$ in adults with abdominal pain]. J Radiol. 2008;89(2):235-243.

2. Lin D, Carlson JJ. Epiploic appendagitis: an often unrecognized cause of abdominal pain. Consultant. 2020;60(3).

3. Subramaniam R. Acute appendagitis: emergency presentation and computed tomographic appearances. Emerg Med J. 2006;23(10):e53.

4. Molla E, Ripolles T, Martinez MJ, Morote V, RoselloSastre E. Primary epiploic appendagitis: US and CT findings. Eur Radiol. 1998;8(3):435-438.

5. Rioux M, Langis P. Primary epiploic appendagitis: clinical, US, and CT findings in 14 cases. Radiology. 1994;191(2):523-526.

6. Chen T, Wu D, Chen H, Yan W, Yang D, Chen G, Ma $\mathrm{K}$, et al. Clinical characteristics of 113 deceased patients with coronavirus disease 2019: retrospective study. BMJ. 2020;368:m1091.

7. Tang N, Li D, Wang X, Sun Z. Abnormal coagulation parameters are associated with poor prognosis in patients with novel coronavirus pneumonia. J Thromb Haemost. 2020;18(4):844-847.

8. Klok FA, Kruip M, van der Meer NJM, Arbous MS,
Gommers D, Kant KM, Kaptein FHJ, et al. Incidence of thrombotic complications in critically ill ICU patients with COVID-19. Thromb Res. 2020;191:145-147.

9. Llitjos JF, Leclerc M, Chochois C, Monsallier JM, Ramakers M, Auvray M, Merouani K. High incidence of venous thromboembolic events in anticoagulated severe COVID-19 patients. J Thromb Haemost. 2020;18(7):17431746.

10. Nahum J, Morichau-Beauchant T, Daviaud F, Echegut P, Fichet J, Maillet JM, Thierry S. Venous thrombosis among critically ill patients with coronavirus disease 2019 (COVID-19). JAMA Netw Open. 2020;3(5):e2010478.

11. Ren B, Yan F, Deng Z, Zhang S, Xiao L, Wu M, Cai L. Extremely high incidence of lower extremity deep venous thrombosis in 48 patients with severe COVID-19 in Wuhan. Circulation. 2020;142(2):181-183.

12. Poissy J, Goutay J, Caplan M, Parmentier E, Duburcq $\mathrm{T}$, Lassalle F, Jeanpierre E, et al. Pulmonary embolism in patients with COVID-19: awareness of an increased prevalence. Circulation. 2020;142(2):184-186.

13. Porfidia A, Pola R. Venous thromboembolism and heparin use in COVID-19 patients: juggling between pragmatic choices, suggestions of medical societies and the lack of guidelines. J Thromb Thrombolysis. 2020;50(1):68-71.

14. Krishnamurthy M, Freedman ML. Complications of anticoagulation with heparin. Virtual Mentor. 2005;7(4):297300 .

15. Devreese KMJ, Linskens EA, Benoit D, Peperstraete H. Antiphospholipid antibodies in patients with COVID-19: A relevant observation? J Thromb Haemost. 2020;18(9):2191-2201.

16. Uthman IW, Gharavi AE. Viral infections and antiphospholipid antibodies. Semin Arthritis Rheum. 2002;31(4):256-263.

17. Galeano-Valle F, Oblitas CM, Ferreiro-Mazon MM, Alonso-Munoz J, Del Toro-Cervera J, di Natale M, DemeloRodriguez P. Antiphospholipid antibodies are not elevated in patients with severe COVID-19 pneumonia and venous thromboembolism. Thromb Res. 2020;192:113-115.

18. Pavoni V, Gianesello L. Antiphospholipid antibodies and thrombosis in severe COVID-19 pneumonia: A difficult relationship. Thromb Res. 2020;194:190-191. 